\title{
Multiple-set split feasibility problems for total asymptotically strict pseudocontractions mappings
}

\author{
LI Yang ${ }^{1 *}$, Shih-Sen Chang ${ }^{2 *}$, Yeol JE Cho ${ }^{3}$ and Jong KYU Kim ${ }^{4}$
}

\author{
* Correspondence: \\ yanglizxs@yahoo.com.cn; \\ changss@yahoo.cn \\ 'Department of Mathematics, \\ South West University of Science \\ and Technology, Mianyang Sichuan \\ 621010, China \\ ${ }^{2}$ College of Statistics and \\ Mathematics, Yunnan University of \\ Finance and Economics, Kunming, \\ Yunnan 650221, China \\ Full list of author information is \\ available at the end of the article
}

\begin{abstract}
The purpose of this article is to propose and investigate an algorithm for solving the multiple-set split feasibility problems for total asymptotically strict pseu-docontractions mappings in infinite-dimensional Hilbert spaces. The results presented in this article improve and extend some recent results of A. Moudafi, H. K. Xu, Y. Censor, A. Segal, T. Elfving, N. Kopf, T. Bortfeld, X. A. Motova, Q. Yang, A. Gibali, S. Reich and others. 2000 AMS Subject Classification: 47J05; 47H09; 49J25.

Keywords: multiple-set split feasibility problem, split feasibility problem, demi-closeness, Opial condition, total asymptotically strict pseudocontraction
\end{abstract}

\section{Introduction and preliminaries}

Throughout this article, we always assume that $H_{1}, H_{2}$ are real Hilbert spaces, “ $\rightarrow$ ", “-” are denoted by strong and weak convergence, respectively, and $F(T)$ is the fixed point set of a mapping $T$.

Let $G$ be a nonempty closed convex subset of $H_{1}$ and $T: G \rightarrow G$ a mapping.

$T$ is said to be a contraction if there exists a constant $\alpha \in(0,1)$ such that

$$
\left\|T_{x}-T_{y}\right\| \leq \alpha\|x-y\|, \quad \forall x, y \in G .
$$

Banach contraction principle guarantees that every contractive mapping defined on complete metric spaces has a unique fixed point.

$T$ is said to be a weak contraction if

$$
\left\|T_{x}-T_{y}\right\| \leq\|x-y\|-\psi(\|x-y\|), \quad \forall x, y \in G .
$$

where $\psi:[0, \infty) \rightarrow[0, \infty)$ is a continuous and nondecreasing function such that $\psi$ is positive on $(0, \infty), \psi(0)=0$, and $\lim _{t \rightarrow \infty} \psi(t)=\infty$. We remark that the class of weak contractions was introduced by Alber and Guerre-Delabriere [1]. In 2001, Rhoades [2] showed that every weak contraction defined on complete metric spaces has a unique fixed point.

$T$ is said to be nonexpansive if

$$
\left\|T_{x}-T_{y}\right\| \leq\|x-y\|, \quad \forall x, y \in G .
$$

\section{Springer}

(C) 2011 Yang et al; licensee Springer. This is an Open Access article distributed under the terms of the Creative Commons Attribution License (http://creativecommons.org/licenses/by/2.0), which permits unrestricted use, distribution, and reproduction in any medium, provided the original work is properly cited. 
$T$ is said to be asymptotically nonexpansive if there exists a sequence $\left\{k_{n}\right\} \subset[1, \infty)$ with $k_{n} \rightarrow 1$ as $n \rightarrow \infty$ such that

$$
\left\|T^{n} x-T^{n} y\right\| \leq \kappa_{n}\|x-y\|, \quad \forall n \geq 1, \quad x, y \in G .
$$

The class of asymptotically nonexpansive mappings was introduced by Goebel and Kirk [3] as a generalization of the class of nonexpansive mappings. They proved that if $G$ is a nonempty closed convex bounded subset of a real uniformly convex Banach space and $T$ is an asymptotically nonexpansive mapping on $G$, then $T$ has a fixed point.

$T$ is said to be total asymptotically nonexpansive if

$$
\left\|T^{n} x-T^{n} y\right\| \leq\|x-y\|+\mu_{n} \phi(\|x-y\|)+\xi_{n}, \quad \forall n \geq 1, \quad x, y \in G .
$$

where $\varphi:[0, \infty) \rightarrow[0, \infty)$ is a continuous and strictly increasing function with $\varphi(0)=$ 0 , and $\left\{\mu_{n}\right\}$ and $\left\{\xi_{n}\right\}$ are nonnegative real sequences such that $\mu_{n} \rightarrow 0$ and $\xi_{n} \rightarrow 0$ as $n \rightarrow \infty$. The class of mapping was introduced by Alber et al. [4]. From the definition, we see that the class of total asymptotically nonexpansive mappings includes the class of asymptotically nonexpansive mappings as special cases, see [5,6] for more details.

$T$ is said to be strictly pseudocontractive if there exists a constant $\kappa \in[0,1)$ such that

$$
\|T x-T y\|^{2} \leq\|x-y\|^{2}+\kappa\|(I-T) x-(I-T) y\|^{2}, \quad \forall x, y \in G .
$$

The class of strict pseudocontractions was introduced by Browder and Petryshyn [7] in a real Hilbert space. In 2007, Marino and Xu [8] obtained a weak convergence theorem for the class of strictly pseudocontractive mappings, see [8] for more details.

$T$ is said to be an asymptotically strict pseudocontraction if there exist a constant $\kappa$ $\in[0,1)$ and a sequence $\left\{k_{n}\right\} \subset[1, \infty)$ with $k_{n} \rightarrow 1$ as $n \rightarrow \infty$ such that

$$
\left\|T^{n} x-T^{n} y\right\|^{2} \leq \kappa_{n}\|x-y\|^{2}+\kappa\left\|\left(I-T^{n}\right) x-\left(I-T^{n}\right) y\right\|^{2}, \quad \forall_{n} \geq 1, \quad x, y \in G .
$$

The class of asymptotically strict pseudocontractions was introduced by Qihou [9] in 1996. Kim and $\mathrm{Xu}$ [10] proved that the class of asymptotically strict pseudocontractions is demiclosed at the origin and also obtained a weak convergence theorem for the class of mappings; see [10] for more details.

In this article, we introduce the following mapping.

Definition 1.1 Let $H$ be a real Hilbert space, and $G$ be a nonempty closed convex subset of $H$. A mapping $T: G \rightarrow G$ is said to be $\left(\kappa,\left\{\mu_{n}\right\},\left\{\xi_{n}\right\}, \varphi\right)$-total asymptotically strict pseudocontractive, if there exists a constant $\kappa \in[0,1)$ and sequences $\left\{\mu_{n}\right\} \subset[0, \infty)$, $\left\{\xi_{n}\right\} \subset[0, \infty)$ with $\mu_{n} \rightarrow 0$ and $\xi_{n} \rightarrow 0$ as $n \rightarrow \infty$, and a continuous and strictly increasing function $\varphi:[0, \infty) \rightarrow[0, \infty)$ with $\varphi(0)=0$ such that

$$
\left\|T^{n} x-T^{n} y\right\|^{2} \leq\|x-y\|^{2}+\kappa\left\|x-y-\left(T^{n} x-T^{n} y\right)\right\|^{2}+\mu_{n} \phi(\|x-y\|)+\xi_{n}, \quad \forall_{n} \geq 1, \quad x, y \in G .
$$

Now, we give an example of total asymptotically strict pseudocontractive mapping.

Let $C$ be a unit ball in a real Hilbert space $l^{2}$ and let $T: C \rightarrow C$ be a mapping defined by

$$
T:\left(x_{1}, x_{2}, \ldots\right) \rightarrow\left(0, x_{1}^{2}, a_{2} x_{2}, a_{3} x_{3}, \ldots\right),
$$


where $\left\{a_{i}\right\}$ is a sequence in $(0,1)$ such that $\prod_{i=2}^{\infty} a_{i}=\frac{1}{2}$.

It is proven in Goebal and Kirk [3] that

(i) $\|T x-T y\| \leq 2\|x-y\|, \quad \forall x, y \in C$;

(ii) $\left\|T^{n} x-T^{n} y\right\| \leq 2 \prod_{j=2}^{n} a_{j}\|x-y\|, \quad \forall x, y \in C, \quad \forall n \geq 2$.

Denote by $\kappa_{1}^{\frac{1}{2}}=2, \kappa_{n}^{\frac{1}{2}}=2 \prod_{j=2}^{n} a_{j}, \quad n \geq 2$, then

$$
\lim _{n \rightarrow \infty} k_{n}=\lim _{n \rightarrow \infty}\left(2 \prod_{j=2}^{n} a_{j}\right)^{2}=1 \text {. }
$$

Letting $\mu_{n}=\left(\kappa_{n}-1\right), \quad \forall n \geq 1, \quad \phi(t)=t^{2}, \quad \forall t \geq 0, \kappa=0$ and $\left\{\xi_{n}\right\}$ be a nonnegative real sequence with $\xi_{n} \rightarrow 0$, then $\forall x, y \in C, \quad n \geq 1$, we have

$$
\left\|T^{n} x-T^{n} y\right\|^{2} \leq\|x-y\|^{2}+\mu_{n} \phi(\|x-y\|)+\kappa \mid\left\|x-y-\left(T^{n} x-T^{n} y\right)\right\|^{2}+\xi_{n} .
$$

Remark 1.2 If $\varphi(\lambda)=\lambda^{2}$ and $\xi_{n}=0$, then total asymptotically strict pseudocontractive mapping is asymptotically strict pseudocontraction mapping.

It is easy to see the following proposition holds.

Proposition 1.3 Let $T: G \rightarrow G$ be a $\left(\kappa,\left\{\mu_{n}\right\},\left\{\xi_{n}\right\}, \varphi\right)$-total asymptotically strict pseudocontractive mapping. If $F(T) \neq \emptyset$, then for each $q \in F(T)$ and for each $x \in G$, the following inequalities hold and are equivalent:

$$
\begin{aligned}
& \left\langle x-q, \quad T^{n} x-q\right\rangle \leq \frac{\kappa+1}{2 k}\|x-q\|^{2}+\frac{\kappa-1}{2 k}\left\|T^{n} x-q\right\|^{2}+\frac{\mu_{n}}{2 \kappa} \phi(\|x-q\|)+\frac{\xi_{n}}{2 \kappa} ; \\
& \left\langle x-T^{n} x, \quad x-q\right\rangle \geq \frac{1-\kappa}{2}\left\|T^{n} x-x\right\|^{2}-\frac{\mu_{n}}{2} \phi(\|x-q\|)-\frac{\xi_{n}}{2} \\
& \left\langle x-T^{n} x, \quad q-T^{n} x\right\rangle \leq \frac{\kappa+1}{2}\left\|T^{n} x-x\right\|^{2}+\frac{\mu_{n}}{2} \phi(\|x-q\|)+\frac{\xi_{n}}{2} .
\end{aligned}
$$

The split feasibility problem (SFP) in finite-dimensional spaces was first introduced by Censor and Elfving [11] for modeling inverse problems which arise from phase retrievals and in medical image reconstruction [12]. Recently, it has been found that the SFP can also be used in various disciplines such as image restoration, computer tomograph, and radiation therapy treatment planning [13-15].

The SFP in an infinite-dimensional Hilbert space can be found in [12,14,16-18].

The purpose of this article is to introduce and study the following multiple-set SFP (MSSFP) for total asymptotically strict pseudocontraction in the framework of infinitedimensional Hilbert spaces:

$$
\text { find } x^{*} \in C \text { such that } A x^{*} \in Q \text {, }
$$

where $A: H_{1} \rightarrow H_{2}$ is a bounded linear operator, $S_{i}: H_{1} \rightarrow H_{1}$ and $T_{i}: H_{2} \rightarrow H_{2}$, $i=1,2, \ldots, N$ are mappings, $C: \bigcap_{i=1}^{N} F\left(S_{i}\right)$ and $Q: \bigcap_{i=1}^{N} F\left(T_{i}\right)$. In the sequel, we use $\Gamma$ to denote the set of solutions of (MSSFP)-(1.12), i.e.,

$$
\Gamma=\{x \in C, \quad A x \in Q\} .
$$


To prove our main results, we first recall some definitions, notations, and conclusions.

Let $E$ be a Banach space. A mapping $T: E \rightarrow E$ is said to be demi-closed at origin, if for any sequence $\left\{x_{n}\right\} \subset E$ with $x_{n} \rightarrow x^{*}$ and $\left\|(I-T) x_{n}\right\| \rightarrow 0$, then $x^{*}=T x^{*}$.

A Banach space $E$ is said to have the Opial property, if for any sequence $\left\{x_{n}\right\}$ with $x_{n} \rightarrow x^{*}$, then

$$
\liminf _{n \rightarrow \infty}\left\|x_{n}-x^{*}\right\|<\liminf _{n \rightarrow \infty}\left\|x_{n}-y\right\|, \quad \forall y \in E \quad \text { with } \quad y \neq x^{*} .
$$

Remark 1.4 It is well known that each Hilbert space possesses the Opial property.

Definition 1.5 Let $H$ bea real Hilbert space.

(1) A mapping $T: H \rightarrow H$ is said to be uniformly $L$-Lipschitzian, if there exists a constant $L>0$, such that

$$
\left\|T^{n} x-T^{n} y\right\| \leq L\|x-y\|, \quad \forall x, y \in H \quad \text { and } n \geq 1 .
$$

(2) A mapping $T: H \rightarrow H$ is said to be semi-compact, if for any bounded sequence $\left\{x_{n}\right\} \subset H$ with $\lim _{n \rightarrow \infty}|| x_{n}-T x_{n} \|=0$, then there exists a subsequence $\left\{x_{n_{i}}\right\} \subset\left\{x_{n}\right\}$ such that $x_{n_{i}}$ converges strongly to some point $x^{*} \in H$.

Lemma 1.6 [10] Let $H$ be a real Hilbert space. If $\left\{x_{n}\right\}$ is a sequence in $H$ weakly convergent to $z$, then

$$
\limsup _{n \rightarrow \infty}\left\|x_{n}-y\right\|^{2}=\limsup _{n \rightarrow \infty}\left\|x_{n}-z\right\|^{2}+\|z-y\|^{2} \quad \forall y \in H .
$$

Proposition 1.7 Assume that $G$ is a closed convex subset of a real Hilbert space $H$ and let $T: G \rightarrow G$ be a $\left(\kappa,\left\{\mu_{n}\right\},\left\{\xi_{n}\right\}, \varphi\right)$-total asymptotically strict pseudocon-traction mapping and uniformly $L$-Lipschitzian. Then the demiclosedness principle holds for $I$ $T$ in the sense that if $\left\{x_{n}\right\}$ is a sequence in $G$ such that $x_{n} \rightarrow x^{*}$, and $\lim \sup _{m \rightarrow \infty} \lim$ $\sup _{n \rightarrow \infty}\left\|x_{n}-T^{m} x_{n}\right\|=0$ then $(I-T) x^{*}=0$. In particular, $x_{n} \rightarrow x^{*}$, and $(I-T) x_{n} \rightarrow 0$ $\Rightarrow(I-T) x^{*}=0$, i.e., $T$ is demiclosed at origin.

Proof Since $\left\{x_{n}\right\}$ is bounded, we can define a function $f$ on $H$ by

$$
f(x)=\limsup _{n \rightarrow \infty}\left\|x_{n}-x\right\|^{2}, \quad \forall x \in H .
$$

By Lemma 1.6, the weak convergence $x_{n} \rightarrow x^{*}$ implies that

$$
f(x)=f\left(x^{*}\right)+\left\|x-x^{*}\right\|^{2}, \quad \forall x \in H .
$$

In particular, for each $m \geq 1$,

$$
f\left(T^{m} x^{*}\right)=f\left(x^{*}\right)+\left\|T^{m} x^{*}-x^{*}\right\|^{2} .
$$


On the other hand, since $T$ is a $\left(\kappa,\left\{\mu_{n}\right\},\left\{\xi_{n}\right\}\right)$-total asymptotically strict pseudocontraction mapping, by (1.8), we get

$$
\begin{aligned}
f\left(T^{m} x^{*}\right) & =\limsup _{n \rightarrow \infty}\left\|x_{n}-T^{m} x^{*}\right\|^{2} \\
& =\limsup _{n \rightarrow \infty}\left\|x_{n}-T^{m} x_{n}+T^{m} x_{n}-T^{m} x^{*}\right\|^{2} \\
& =\limsup _{n \rightarrow \infty}\left(\left\|x_{n}-T^{m} x_{n}\right\|^{2}+2\left\langle x_{n}-T^{m} x_{n}, T^{m} x_{n}-T^{m} x^{*}\right\rangle+\left\|T^{m} x_{n}-T^{m} x^{*}\right\|^{2}\right) \\
& \leq \limsup _{n \rightarrow \infty}\left\|x_{n}-T^{m} x_{n}\right\|\left(\left\|x_{n}-T^{m} x_{n}\right\|+2 L\left\|x_{n}-x^{*}\right\|\right) \\
& +\limsup _{n \rightarrow \infty}\left(\left\|x_{n}-x^{*}\right\|^{2}+k\left\|x_{n}-T^{m} x_{n}-\left(x^{*}-T^{m} x^{*}\right)\right\|^{2}+\mu_{m} \phi\left(\left\|x_{n}-x^{*}\right\|\right)+\xi_{m}\right)
\end{aligned}
$$

Taking $\lim \sup _{m \rightarrow \infty}$ on both sides and observing the facts that $\lim _{m \rightarrow \infty} \mu_{m}=0$, $\lim _{m \rightarrow \infty} \xi_{m}=0$ and $\lim \sup _{m \rightarrow \infty} \lim \sup _{n \rightarrow \infty}|| x_{n}-T^{m} x_{n} \|=0$, we derive that

$$
\limsup _{m \rightarrow \infty} f\left(T^{m} x^{*}\right) \leq \limsup _{n \rightarrow \infty}\left\|x_{n}-x^{*}\right\|^{2}+k \limsup _{m \rightarrow \infty}\left\|x^{*}-T^{m} x^{*}\right\|^{2}
$$

Since $\lim \sup _{m \rightarrow \infty} f\left(T^{m} x^{*}\right)=f\left(x^{*}\right)+\lim \sup _{m \rightarrow \infty}\left\|T^{m} x^{*}-x^{*}\right\|^{2}$, and $f\left(x^{*}\right)=\lim \sup _{\mathrm{n} \rightarrow \infty}$ $\left\|x_{n}-x^{*}\right\|^{2}$, it follows from (1.15) that $\lim _{\sup } \operatorname{sum}_{m \rightarrow \infty}\left\|x^{*}-T^{m} x^{*}\right\|^{2}=0$. That is, $T^{m} x^{*} \rightarrow$ $x^{*}$; hence $T x^{*}=x^{*}$.

Lemma 1.8 [19] Let $\left\{a_{n}\right\},\left\{b_{n}\right\}$ and $\left\{\delta_{n}\right\}$ be sequences of nonnegative real numbers satisfying

$$
a_{n+1} \leq\left(1+\delta_{n}\right) a_{n}+b_{n}, \quad \forall n \geq 1 .
$$



\section{Multiple-set split feasibility problem}

For solving the multiple-set split feasibility problem (1.12), let us assume that the following conditions are satisfied:

1. $H_{1}$ and $H_{2}$ are two real Hilbert spaces, $A: H_{1} \rightarrow H_{2}$ is a bounded linear operator;

2. Let $G, \tilde{G}$ be a nonempty closed convex subset of $H_{1}$ and $H_{2}$ respectively, $S_{i}: G$ $\rightarrow G, i=1,2, \ldots, N$, is a uniformly $L_{i}$-Lipschitzian and $\left(\beta_{i},\left\{\mu_{i, n}\right\},\left\{\xi_{i, n}\right\}, \varphi_{i}\right)$-total asymptotically strictly pseudocontractive mapping and $T_{i}: \tilde{G} \rightarrow \tilde{G}_{,} \quad i=1,2, \ldots, N$, is a uniformly $\tilde{L}_{i}$-Lipschitzian and $\left(k_{i}\left\{\tilde{\mu}_{i, n}\right\},\left\{\tilde{\xi}_{i, n}\right\}, \tilde{\phi}_{i}\right)$-total asymptotically strictly pseudocontractive mapping which satisfy the following conditions:

(i) $C: \bigcap_{i=1}^{N} F\left(S_{i}\right) \neq \emptyset, \quad Q:=\bigcap_{i=1}^{N} F\left(T_{i}\right) \neq \emptyset$;

(ii) $\beta=\max _{1 \leq i \leq N} \quad \beta_{i}<1, \quad \kappa=\max _{1 \leq i \leq N} \quad \kappa_{i}<1$;

(iii) $L:=\max _{1 \leq i \leq N} L_{i}<\infty, \quad \tilde{L}:=\max _{1 \leq i \leq N} \quad \tilde{L}_{i}<\infty$;

(iv) $\mu_{n}=\max _{1 \leq i \leq N}\left\{\mu_{i, n}, \tilde{\mu}_{i, n}\right\}, \xi_{n}=\max _{1 \leq i \leq N}\left\{\xi_{i, n}, \tilde{\xi}_{i, n}\right\}$ and $\sum_{i=1}^{\infty} \mu_{n}<\infty, \sum_{i=1}^{\infty} \xi_{n}<\infty$.

(v) $\phi=\max _{1 \leq i \leq N}\left\{\phi_{i},\left\{\tilde{\phi}_{i}\right\}\right.$ 
We are now in a position to give the following result:

Theorem 2.1 Let $H_{1}, H_{2}, G, \tilde{G}, A,\left\{S_{i}\right\},\left\{T_{i}\right\}, C, Q, \beta, \kappa, L, \tilde{L},\left\{\mu_{n}\right\},\left\{\xi_{n}\right\}$ and $\varphi$ be the same as above. In addition, there exist positive constants $M$ and $M^{*}$ such that $\varphi$ $(\lambda) \leq M^{*} \lambda^{2}$ for all $\lambda \geq M$. Let $\left\{x_{n}\right\}$ be the sequence generated by:

$$
\left\{\begin{array}{l}
x_{1} \in G \quad \text { chosen arbitrarily } \\
x_{n+1}=\left(1-\alpha_{n}\right) u_{n}+\alpha_{n} S_{n}^{n}\left(u_{n}\right), \\
u_{n}=x_{n}+\gamma A^{*}\left(T_{n}^{n}-I\right) A x_{n}, \quad \forall n \geq 1,
\end{array}\right.
$$

where $S_{n}^{n}=S_{n(\bmod N)^{\prime}}^{n} \quad T_{n}^{n}=T_{n(\bmod N)}^{n} \quad \forall n \geq 1, \quad\left\{\alpha_{n}\right\}$ is a sequence in $[0,1]$ and $\gamma$ $>0$ is a constant satisfying the following conditions:

(vi) $\alpha_{n} \in(\delta, 1-\beta), \quad \forall n \geq 1$ and $\gamma \in\left(0, \frac{1-\kappa}{\|A\|^{2}}\right)$, where $\delta \in(0,1-\beta)$ is a positive constant.

(I) If $\Gamma \neq \emptyset$ (where $\Gamma$ is the set of solutions to (MSSFP)-(1.12)), then $\left\{x_{n}\right\}$ converges weakly to a point $x^{*} \in \Gamma$.

(II) In addition, if there exists a positive integer $j$ such that $S_{j}$ is semi-compact, then $\left\{x_{n}\right\}$ and $\left\{u_{n}\right\}$ both converge strongly to $x^{*} \in \Gamma$.

\section{The proof of conclusion (I)}

(1) First we prove that for each $p\lfloor\Gamma$, the following limits exist

$$
\lim _{n \rightarrow \infty}\left\|x_{n}-p\right\| \quad \text { and } \quad \lim _{n \rightarrow \infty}\left\|u_{n}-p\right\| \text {. }
$$

In fact, since $\varphi$ is an increasing function, it results that $\varphi(\lambda) \leq \varphi(M)$, if $\lambda \leq M$ and $\varphi(\lambda) \leq M^{*} \lambda^{2}$, if $\lambda \geq M$. In either case, we can obtain that

$$
\phi(\lambda) \leq \phi(M)+M^{*} \lambda^{2}, \quad \forall \lambda \geq 0
$$

Since $p \in \Gamma$, then $p \in C:=\bigcap_{i=1}^{N} F\left(S_{i}\right)$ and $A p \in Q:=\bigcap_{i=1}^{N} F\left(T_{i}\right)$. From (2.1) and (1.10) we have

$$
\begin{aligned}
\left\|x_{n+1}-p\right\|^{2}= & \left\|u_{n}-p-\alpha_{n}\left(u_{n}-S_{n}^{n} u_{n}\right)\right\|^{2} \\
= & \left\|u_{n}-p\right\|^{2}-2 \alpha_{n}\left\langle u_{n}-p, u_{n}-S_{n}^{n} u_{n}\right\rangle+\alpha_{n}^{2}\left\|u_{n}-S_{n}^{n} u_{n}\right\|^{2} \\
\leq & \left\|u_{n}-p\right\|^{2}-\alpha_{n}(1-\beta)\left\|u_{n}-S_{n}^{n} u_{n}\right\|^{2} \\
& +\alpha_{n} \mu_{n} \phi\left(\left\|u_{n}-p\right\|\right)+\alpha_{n} \xi_{n}+\alpha_{n}^{2}\left\|u_{n}-S_{n}^{n} u_{n}\right\|^{2}(b y(1.10)) \\
\leq & \left\|u_{n}-p\right\|^{2}-\alpha_{n}\left(1-\beta-\alpha_{n}\right)\left\|u_{n}-S_{n}^{n} u_{n}\right\|^{2} \\
& +\alpha_{n} \mu_{n}\left(\phi(M)+M^{*}\left(\left\|u_{n}-p\right\|\right)^{2}\right)+\alpha_{n} \xi_{n} \\
= & \left(1+\alpha_{n} \mu_{n} M^{*}\right)\left\|u_{n}-p\right\|^{2}-\alpha_{n}\left(1-\beta-\alpha_{n}\right)\left\|u_{n}-S_{n}^{n} u_{n}\right\|^{2} \\
& +\alpha_{n} \mu_{n} \phi(M)+\alpha_{n} \xi_{n}
\end{aligned}
$$

On the other hand, since

$$
\begin{aligned}
\left\|u_{n}-p\right\|^{2} & =\left\|x_{n}-p+\gamma A^{*}\left(T_{n}^{n}-I\right) A x_{n}\right\|^{2} \\
& =\left\|x_{n}-p\right\|^{2}+\gamma^{2}\left\|A^{*}\left(T_{n}^{n}-I\right) A x_{n}\right\|^{2}+2 \gamma\left\langle x_{n}-p, A^{*}\left(T_{n}^{n}-I\right) A x_{n}\right\rangle,
\end{aligned}
$$


and

$$
\begin{aligned}
\left\|A^{*}\left(T_{n}^{n}-I\right) A x_{n}\right\|^{2} & =\left\langle A^{*}\left(T_{n}^{n}-I\right) A x_{n}, A^{*}\left(T_{n}^{n}-I\right) A x_{n}\right\rangle \\
& =\left\langle A A^{*}\left(T_{n}^{n}-I\right) A x_{n}, \quad\left(T_{n}^{n}-I\right) A x_{n}\right\rangle \\
& \leq\|A\|^{2}\left\|T_{n}^{n} A x_{n}-A x_{n}\right\|^{2},
\end{aligned}
$$

It follows from (1.11) we have

$$
\begin{aligned}
& \left\langle x_{n}-p, A^{*}\left(T_{n}^{n}-I\right) A x_{n}\right\rangle=\left\langle A x_{n}-A p, \quad\left(T_{n}^{n}-I\right) A x_{n}\right\rangle \\
& =\left\langle\left(A x_{n}-A p\right)+\left(T_{n}^{n}-I\right) A x_{n}-\left(T_{n}^{n}-I\right) A x_{n}, \quad\left(T_{n}^{n}-I\right) A x_{n}\right\rangle \\
& =\left\langle T_{n}^{n} A x_{n}-A p, \quad T_{n}^{n} A x_{n}-A x_{n}\right\rangle-\left\|\left(T_{n}^{n}-I\right) A x_{n}\right\|^{2} . \\
& \leq \frac{1+\kappa}{2}\left\|\left(T_{n}^{n}-I\right) A x_{n}\right\|^{2}+\frac{\mu_{n}}{2} \phi\left(\left\|A x_{n}-A p\right\|\right)+\frac{\xi n}{2}-\left\|\left(T_{n}^{n}-I\right) A x_{n}\right\|^{2} . \\
& \leq \frac{\kappa-1}{2}\left\|\left(T_{n}^{n}-I\right) A x_{n}\right\|^{2}+\frac{\mu_{n}}{2}\left(\phi(M)+M^{*}\left\|A x_{n}-A p\right\|^{2}\right)+\frac{\xi_{n}}{2} . \\
& \leq \frac{\kappa-1}{2}\left\|\left(T_{n}^{n}-I\right) A x_{n}\right\|^{2}+\frac{\mu_{n}}{2} M^{*}\left\|A x_{n}-A p\right\|^{2}+\frac{\mu_{n}}{2} \phi(M)+\frac{\xi_{n}}{2} .
\end{aligned}
$$

Substituting (2.6) and (2.7) into (2.5) and simplifying it, we have

$$
\begin{aligned}
\left\|u_{n}-p\right\|^{2} \leq & \left\|x_{n}-p\right\|^{2}+\gamma^{2}\|A\|^{2}\left\|T_{n}^{n} A x_{n}-A x_{n}\right\|^{2}+\gamma(\kappa-1)\left\|\left(T_{n}^{n}-I\right) A x_{n}\right\|^{2} \\
& +\gamma \mu_{n} M^{*}\left\|A x_{n}-A p\right\|^{2}+\gamma \mu_{n} \phi(M)+\gamma \xi_{n} \\
= & \left\|x_{n}-p\right\|^{2}-\gamma\left(1-\kappa-\gamma\|A\|^{2}\right)\left\|T_{n}^{n} A x_{n}-A x_{n}\right\|^{2} \\
& +\gamma \mu_{n} M^{*}\left\|A x_{n}-A p\right\|^{2}+\gamma \mu_{n} \phi(M)+\gamma \xi_{n} \\
\leq & \left(1+\gamma \mu_{n} M^{*}\|A\|^{2}\right)\left\|x_{n}-p\right\|^{2}-\gamma\left(1-\kappa-\gamma\|A\|^{2}\right)\left\|T_{n}^{n} A x_{n}-A x_{n}\right\|^{2} \\
& +\gamma \mu_{n} \phi(M)+\gamma \xi_{n}
\end{aligned}
$$

Substituting (2.8) into (2.4) and after simplifying we have

$$
\begin{aligned}
\left\|x_{n+1}-p\right\|^{2} & \leq\left(1+\alpha_{n} \mu_{n} M^{*}\right)\left\{\left(1+\gamma \mu_{n} M^{*}\|A\|^{2}\right)\left\|x_{n}-p\right\|^{2}\right. \\
& \left.-\gamma\left(1-\kappa-\gamma\|A\|^{2}\right)\left\|T_{n}^{n} A x_{n}-A x_{n}\right\|^{2}+\gamma \mu_{n} \phi(M)+\gamma \xi_{n}\right\} \\
& -\alpha_{n}\left(1-\beta-\alpha_{n}\right)\left\|u_{n}-S_{n}^{n} u_{n}\right\|^{2}+\alpha_{n} \mu_{n} \phi(M)+\alpha_{n} \xi_{n} \\
\leq & \left(1+\delta_{n}\right)\left\|x_{n}-p\right\|^{2}-\gamma\left(1-\kappa-\gamma\|A\|^{2}\right)\left\|T_{n}^{n} A x_{n}-A x_{n}\right\|^{2} \\
& -\alpha_{n}\left(1-\beta-\alpha_{n}\right)\left\|u_{n}-S_{n}^{n} u_{n}\right\|^{2}+b_{n}
\end{aligned}
$$

where

$$
\begin{aligned}
& \delta_{n}=\alpha_{n} \mu_{n} M^{*}+\gamma \mu_{n} M^{*}\|A\|^{2}+\gamma\|A\|^{2} \alpha_{n} \mu_{n}^{2}\left(M^{*}\right)^{2} \\
& b_{n}=\left(\left(1+\alpha_{n} \mu_{n} M^{*}\right) \gamma+\alpha_{n}\right) \mu_{n} \phi(M)+\left(\left(1+\alpha_{n} \mu_{n} M^{*}\right) \gamma+\alpha_{n}\right) \xi_{n}
\end{aligned}
$$

By condition (vi) we have

$$
\left\|x_{n+1}-p\right\|^{2} \leq\left(1+\delta_{n}\right)\left\|x_{n}-p\right\|^{2}+b_{n}
$$

By condition (iv), $\sum_{n=1}^{\infty} \delta_{n}<\infty$ and $\sum_{n=1}^{\infty} b_{n}<\infty$. Hence, from Lemma 1.8 we know that the following limit exists

$$
\lim _{n \rightarrow \infty}\left\|x_{n}-p\right\|
$$


Consequently, from (2.9) and (2.10) we have that

$$
\begin{aligned}
& \gamma\left(1-\kappa-\gamma\|A\|^{2}\right)\left\|\left(T_{n}^{n}-I\right) A x_{n}\right\|^{2}+\alpha_{n}\left(1-\beta-\alpha_{n}\right)\left\|u_{n}-S_{n}^{n} u_{n}\right\|^{2} \\
& \leq\left\|x_{n}-p\right\|^{2}-\left\|x_{n+1}-p\right\|^{2}+\delta_{n}\left\|x_{n}-p\right\|^{2}+b_{n} \rightarrow 0(\text { as } n \rightarrow \infty) .
\end{aligned}
$$

This together with the condition (vi) implies that

$$
\lim _{n \rightarrow \infty}\left\|u_{n}-S_{n}^{n} u_{n}\right\|=0
$$

and

$$
\lim _{n \rightarrow \infty}\left\|\left(T_{n}^{n}-I\right) A x_{n}\right\|=0 .
$$

It follows from (2.5), (2.10) and (2.12) that the limit $\left\|u_{n}-p\right\|$ exists.

The conclusion (1) is proved.

\section{(2) Next we prove that}

$$
\lim _{n \rightarrow \infty}\left\|x_{n+1}-x_{n}\right\|=0 \text { and } \lim _{n \rightarrow \infty}\left\|u_{n+1}-u_{n}\right\|=0 .
$$

In fact, it follows from (2.1) that

$$
\begin{aligned}
\left\|x_{n+1}-x_{n}\right\| & =\left\|\left(1-\alpha_{n}\right) u_{n}+\alpha_{n} S_{n}^{n}\left(u_{n}\right)-x_{n}\right\| \\
& =\left\|\left(1-\alpha_{n}\right)\left(x_{n}+\gamma A^{*}\left(T_{n}^{n}-I\right) A x_{n}\right)+\alpha_{n} S_{n}^{n}\left(u_{n}\right)-x_{n}\right\| \\
& =\left\|\left(1-\alpha_{n}\right) \gamma A^{*}\left(T_{n}^{n}-I\right) A x_{n}+\alpha_{n}\left(S_{n}^{n}\left(u_{n}\right)-x_{n}\right)\right\| \\
& =\left\|\left(1-\alpha_{n}\right) \gamma A^{*}\left(T_{n}^{n}-I\right) A x_{n}+\alpha_{n}\left(S_{n}^{n}\left(u_{n}\right)-u_{n}\right)+\alpha_{n}\left(u_{n}-x_{n}\right)\right\| \\
& =\left\|\left(1-\alpha_{n}\right) \gamma A^{*}\left(T_{n}^{n}-I\right) A x_{n}+\alpha_{n}\left(S_{n}^{n}\left(u_{n}\right)-u_{n}\right)+\alpha_{n} \gamma A^{*}\left(T_{n}^{n}-I\right) A x_{n}\right\| \\
& =\left\|\gamma A^{*}\left(T_{n}^{n}-I\right) A x_{n}+\alpha_{n}\left(S_{n}^{n}\left(u_{n}\right)-u_{n}\right)\right\| .
\end{aligned}
$$

In view of (2.11) and (2.12) we have that

$$
\lim _{n \rightarrow \infty}\left\|x_{n+1}-x_{n}\right\|=0 \text {. }
$$

Similarly, it follows from (2.1), (2.12), and (2.14) that

$$
\begin{aligned}
\left\|u_{n+1}-u_{n}\right\|= & \left\|x_{n+1}+\gamma A^{*}\left(T_{n+1}^{n+1}-I\right) A x_{n+1}-\left(x_{n}+\gamma A^{*}\left(T_{n}^{n}-I\right) A x_{n}\right)\right\| \\
\leq & \left\|x_{n+1}-x_{n}\right\|+\gamma\left\|A^{*}\left(T_{n+1}^{n+1}-I\right) A x_{n+1}\right\| \\
& +\gamma\left\|A^{*}\left(T_{n}^{n}-I\right) A x_{n}\right\| \rightarrow 0(\text { as } n \rightarrow \infty) .
\end{aligned}
$$

The conclusion (2.13) is proved.

(3) Next we prove that for each $j=1,2, \ldots, N-1$,

$$
\left\|u_{i N+j}-S_{j} u_{i N+j}\right\| \rightarrow 0 \text { and }\left\|A x_{i N+j}-T_{j} A x_{i N+j}\right\| \rightarrow 0 \quad(\text { as } i \rightarrow \infty),
$$

In fact, from (2.11) we have

$$
\eta_{i N+j}:=\left\|u_{i N+j}-S_{j}^{i N+j} u_{i N+j}\right\| \rightarrow 0 \quad(\text { as } i \rightarrow \infty) .
$$


Since $S_{j}$ is uniformly $L_{j}$-Lipschitzian continuous, it follows from (2.13) and (2.17) that

$$
\begin{aligned}
\| u_{i N+j}- & S_{j} u_{i N+j}\|=\| u_{i N+j}-S_{j}^{i N+j} u_{i N+j}\|+\| S_{j}^{i N+j} u_{i N+j}-S_{j} u_{i N+j} \| \\
\leq & \eta_{i N+j}+L_{j}\left\|S_{j}^{i N+j-1} u_{i N+j}-u_{i N+j}\right\| \\
\leq & \eta_{i N+j}+L_{j}\left\{\left\|S_{j}^{i N+j-1} u_{i N+j}-S_{j}^{i N+j-1} u_{i N+j-1}\right\|\right. \\
& \left.+L_{j}\left\|S_{j}^{i N+j-1} u_{i N+j-1}-u_{i N+j}\right\|\right\} \\
\leq & \eta_{i N+j}+L_{j}^{2}\left\|u_{i N+j}-u_{i N+j-1}\right\| \\
& +L_{j}\left\|S_{j}^{i N+j-1} u_{i N+j-1}-u_{i N+j-1}+u_{i N+j-1}-u_{i N+j}\right\| \\
\leq & \left.\eta_{i N+j}+L_{j}\left(1+L_{j}\right)\left\|u_{i N+j}-u_{i N+j-1}\right\|+L_{j} \eta_{i N+j-1} \rightarrow 0 \quad \text { as } i \rightarrow \infty\right)
\end{aligned}
$$

Similarly, for each $j=1,2, \ldots, N-1$, from (2.13) we have

$$
\varsigma_{i N+j}:=\left\|A x_{i N+j}-T_{j}^{i N+j} A x_{i N+j}\right\| \rightarrow 0 \quad(\text { as } i \rightarrow \infty) .
$$

Since $T_{j}$ is uniformly $\tilde{L}_{j}$-Lipschitzian continuous, by the same way as above, from (2.13) and (2.18), we can also prove that

$$
\left\|A x_{i N+j}-T_{j} A x_{i N+j}\right\| \rightarrow 0 \quad(\text { as } i \rightarrow \infty) .
$$

(4) Finally we prove that $x_{n} \rightarrow x^{*}$ and $u_{n} \rightarrow x^{*}$ which is a solution of (MSSFP)(1.12).

Since $\left\{u_{n}\right\}$ is bounded. There exists a subsequence $\left\{u_{n_{i}}\right\} \subset\left\{u_{n}\right\}$ such that $u_{n_{i}} \rightarrow x^{*}$ (some point in $H_{1}$ ). Hence, for any positive integer $j=1,2, \ldots, N$, there exists a subsequence $\left\{n_{i}(j)\right\} \subset\left\{n_{i}\right\}$ with $n_{i}(j)(\bmod N)=j$ such that $u_{n_{i}(j)} \rightarrow x^{*}$. Again from $(2.16)$ we have

$$
\left\|u_{n_{i}(j)}-S_{j} u_{n_{i}(j)}\right\| \rightarrow 0 \quad\left(\text { as } n_{i}(j) \rightarrow \infty\right)
$$

Since $S_{j}$ is demiclosed at zero (see Proposition 1.7), it gets that $x^{*} \in F\left(S_{j}\right)$. By the arbitrariness of $j=1,2, \ldots, N$, we have $x^{*} \in C:=\bigcap_{j=1}^{N} F\left(S_{j}\right)$.

Moreover, from (2.1) and (2.12) we have

$$
x_{n_{i}}=u_{n_{i}}-\gamma A^{*}\left(T_{n_{i}}^{n_{i}}-I\right) A x_{n_{i}} \rightarrow x^{*} .
$$

Since $A$ is a linear bounded operator, it gets $A x_{n_{i}} \rightarrow A x^{*}$. For any positive integer $k=$ $1,2, \ldots, N$, there exists a subsequence $\left\{n_{i}(k)\right\} \subset\left\{n_{i}\right\}$ with $n_{i}(k)(\bmod N)=k$ such that $A x_{n_{i}(k)} \rightarrow A x^{*}$. In view of (2.16) we have

$$
\left\|A x_{n_{i}(k)}-T_{k} A x_{n_{i}(k)}\right\| \rightarrow 0 \quad\left(\text { as } n_{i}(k) \rightarrow \infty\right) .
$$

Since $T_{k}$ is demiclosed at zero, we have $A x^{*} \in F\left(T_{k}\right)$. By the arbitrariness of $k=1$, $2, \ldots, N$, it yields $A x^{*} \in Q:=\bigcap_{k=1}^{N} F\left(T_{k}\right)$. This together with $x^{*} \in C$ shows that $x^{*} \in \Gamma$, i. e., $x^{*}$ is a solution to the (MSSFP)-(1.12).

Now we prove that $x_{n} \rightarrow x^{*}$ and $u_{n} \rightarrow x^{*}$. 
In fact, if there exists another subsequence $\left\{u_{n_{i}}\right\} \subset\left\{u_{n}\right\}$ such that $u_{n_{i}(j)} \rightarrow \gamma^{*} \in \Gamma$ with $y^{*} \neq x^{* *}$. Consequently, by virtue of (2.2) and the Opial property of Hilbert space, we have

$$
\begin{aligned}
\liminf _{n_{i} \rightarrow \infty}\left\|u_{n_{i}}-x^{*}\right\| & <\liminf _{n_{i} \rightarrow \infty}\left\|u_{n_{i}}-y^{*}\right\|=\lim _{n \rightarrow \infty}\left\|u_{n}-y^{*}\right\| \\
& =\liminf _{n_{i} \rightarrow \infty}\left\|u_{n_{i}}-y^{*}\right\|<\lim _{n_{j} \rightarrow \infty}\left\|u_{n_{j}}-x^{*}\right\| \\
& =\liminf _{n \rightarrow \infty}\left\|u_{n}-x^{*}\right\|=\lim _{n_{i} \rightarrow \infty}\left\|u_{n_{i}}-x^{*}\right\| .
\end{aligned}
$$

This is a contradiction. Therefore, $u_{n} \rightarrow x^{*}$. By using (2.1) and (2.12), we have

$$
x_{n}=u_{n}-\lambda A^{*}\left(T_{n}^{n}-I\right) A x_{n} \rightarrow x^{*} .
$$

\section{The proof of conclusion (II).}

Without loss of generality, we can assume that $S_{1}$ is semi-compact. It follows from (2.20) that

$$
\left\|u_{n_{i}(1)}-S_{1} u_{n_{i}(1)}\right\| \rightarrow 0 \quad\left(\text { as } n_{i}(1) \rightarrow \infty\right)
$$

Therefore, there exists a subsequence of $\left\{u_{n_{i}(1)}\right\}$ (for the sake of convenience we still denote it by $\left\{u_{n_{i}(1)}\right\}$ such that $u_{n_{i}(1)} \rightarrow u^{*} \in H_{1}$ (some point in $H_{1}$ ). Since $u_{n_{i}(1)} \rightarrow x^{*}$. This implies that $x^{*}=u^{*}$, and so $u_{n_{i}(1)} \rightarrow x^{*} \in \Gamma$. By virtue of (2.2) we know that $\lim _{n \rightarrow \infty}\left\|u_{n}-x^{*}\right\|=0$ and $\lim _{n \rightarrow \infty}\left\|x_{n}-x^{*}\right\|=0$, i.e., $\left\{u_{n}\right\}$ and $\left\{x_{n}\right\}$ both converge strongly to $x^{*} \in \Gamma$.

This completes the proof of Theorem 2.1.

Remark 2.2 Since the class of total asymptotically strict pseudocontractive mappings includes the class of asymptotically strict pseudocontractions mappings and the class of strict pseudocontractions mappings as special cases, Theorem 2.1 improves and extend the corresponding results of Censor et al. [14,15], Yang [17], Moudafi [20], Xu [21], Censor and Segal [22], Censor et al. [23] and others.

\section{Acknowledgements}

The authors would like to thank the referees for useful comments and suggestions. This study was supported by the Natural Science Foundation of Sichuan Province (No. 08ZA008).

\footnotetext{
Author details

${ }^{1}$ Department of Mathematics, South West University of Science and Technology, Mianyang Sichuan 621010, China ${ }^{2}$ College of Statistics and Mathematics, Yunnan University of Finance and Economics, Kunming, Yunnan 650221, China ${ }^{3}$ Department of Mathematics Education, Rins Gyeongsang National University, Jinju 660-701, Korea ${ }^{4}$ Department of Mathematics Education, Kyungnam University Masan, Kyungnam 631-701, Korea
}

\section{Authors' contributions}

All authors have read and approved the final manuscript.

\section{Competing interests}

The authors declare that they have no competing interests.

1. Alber, Yal, Guerre-Delabriere, S: On the projection methods for fixed point problems. Analysis. 21, 17-39 (2001)

2. Rhoades, BE: Some theorems on weakly contractive maps. Nonlinear Anal: Theory Methods Appl. 47, 2683-2693 (2001). doi:10.1016/S0362-546X(01)00388-1

3. Goebel, K, Kirk, WA: A fixed point theorem for asymptotically nonexpansive mappings. Proc Am Math Soc. 35, 171-174 (1972). doi:10.1090/S0002-9939-1972-0298500-3

4. Alber, Yal, Chidume, CE, Zegeye, H: Approximating fixed points of total asymptotically nonex-pansive mappings. Fixed Point Theory Appl. 10673, 20 (2006) 
5. Chidume, CE, Ofoedu, EU: A new iteration process for approximation of common fixed points for finite families of total asymptotically nonexpansive mappings. Int J Math Math Sci. 615107, 17 (2009)

6. Chidume, CE, Ofoedu, EU: Approximation of common fixed points for finite families of total asymptotically nonexpansive mappings. J Math Anal Appl. 333, 128-141 (2007). doi:10.1016/j.jmaa.2006.09.023

7. Browder, FE, Petryshyn, WV: Construction of fixed points of nonlinear mappings in Hilbert space. J Math Anal Appl. 20, 197-228 (1967). doi:10.1016/0022-247X(67)90085-6

8. Marino, G, Xu, HK: Weak and strong convergence theorems for strict pseudo-contractions in Hilbert spaces. J Math Anal Appl. 329, 336-346 (2007). doi:10.1016/j.jmaa.2006.06.055

9. Qihou, L: Convergence theorems of the sequence of iterates for asymptotically demicontractive and hemicontractive mappings. Nonlinear Anal: Theory Methods Appl. 26, 1835-1842 (1996). doi:10.1016/0362-546X(94)00351-H

10. Kim, TH, Xu, HK: Convergence of the modified Mann's iteration method for asymptotically strict pseudo-contractions. Nonlinear Anal: Theory Methods Appl. 68, 2828-2836 (2008). doi:10.1016/j.na.2007.02.029

11. Censor, Y, Elfving, T: A multi-projection algorithm using Bregman projections in a product space. Numer Algorithms. 8, 221-239 (1994). doi:10.1007/BF02142692

12. Byrne, C: Iterative oblique projection onto convex subsets and the split feasibility problem. Inverse Problem. 18 441-453 (2002). doi:10.1088/0266-5611/18/2/310

13. Censor, Y, Bortfeld, T, Martin, B, Trofimov, A: A unified approach for inversion problem in intensity-modulated radiation therapy. Phys Med Biol. 51, 2353-2365 (2006). doi:10.1088/0031-9155/51/10/001

14. Censor, Y, Elfving, T, Kopf, N, Bortfeld, T: The multiple-sets split feasibility problem and its applications. Inverse Problem. 21, 2071-2084 (2005). doi:10.1088/0266-5611/21/6/017

15. Censor, Y, Motova, XA, Segal, A: Pertured projections and subgradient projections for the multiple-setssplit feasibility problem. J Math Anal Appl. 327, 1244-1256 (2007). doi:10.1016/j.jmaa.2006.05.010

16. Xu, HK: A variable Krasnosel'skii-Mann algorithm and the multiple-sets split feasibility problem. Inverse Problem. 22, 2021-2034 (2006). doi:10.1088/0266-5611/22/6/007

17. Yang, Q: The relaxed CQ algorithm for solving the split feasibility problem. Inverse Problem. 20, 1261-1266 (2004). doi:10.1088/0266-5611/20/4/014

18. Zhao, J, Yang, Q: Several solution methods for the split feasibility problem. Inverse Problem. 21, 1791-1799 (2005). doi:10.1088/0266-5611/21/5/017

19. Aoyama, K, Kimura, W, Takahashi, W, Toyoda, M: Approximation of common fixed points of acountable family of nonexpansive mappings on a Banach space. Nonlinear Anal: Theory Methods Appl. 67(8), 2350-2360 (2007). doi:10.1016/j.na.2006.08.032

20. Moudafi, A: The split common fixed point problem for demi-contractive mappings. Inverse Prob-lem. 26(055007), 6 (2010)

21. Xu, HK: Iterative methods for split feasibility problem in infinite-dimensional Hilbert spaces. Inverse Problem. 26(105018), 17 (2010)

22. Censor, Y, Segal, A: The split common fixed point problem for directed operators. J Convex Anal. 16, 587-600 (2009)

23. Censor, Y, Gibali, A, Reich, S: Algorithms for the split variational inequality problem. Numer Algorithm (accepted)

doi:10.1186/1687-1812-2011-77

Cite this article as: Yang et al: Multiple-set split feasibility problems for total asymptotically strict pseudocontractions mappings. Fixed Point Theory and Applications 2011 2011:77.

\section{Submit your manuscript to a SpringerOpen ${ }^{\circ}$ journal and benefit from:}

- Convenient online submission

Rigorous peer review

- Immediate publication on acceptance

- Open access: articles freely available online

- High visibility within the field

- Retaining the copyright to your article

Submit your next manuscript at $\boldsymbol{\nabla}$ springeropen.com 